gegenüber: die „positive“ Deutung der „politischen Gesellschaft" als eines umfassenden Ganzen sowie die kritische Perspektive auf die „defizitären“ Effekte individualisierter und fragmentierter Vergesellschaftung (150f.).

Auch in der Rekonstruktion von Arendts politischer Hermeneutik gelingen Sigwart begriffliche Vereindeutigungen: Ihre performative Perspektive auf die Konstituierung der „Wir“-Gemeinschaft durch das Zusammenspiel von kreativen Praxen politischer Akteure und der diese Praxen interpretierenden Theoretiker wird als „plurale Subjektivität" einerseits von Heideggers pejorativer Bezeichnung des anonymen "Man“ und andererseits von Habermas' „intersubjektivistischer“ Auflösung politischen Handelns abgegrenzt (414ff., 477ff.). Aber während Deweys politische Hermeneutik treffend "als immanente politische Hermeneutik" bezeichnet wird, bleibt die Etikettierung von Arendts politische Theorie als „offene politische Hermeneutik " weniger eindeutig (312f.). Was Sigwart damit auf den Begriff bringen will, ist Arendts Unentschiedenheit hinsichtlich des normativ-kritischen Status einer hermeneutischen politischen Theorie. Denn wiewohl Arendt den Standpunkt eines privilegierten politisch-philosophischen Blicks auf politische Praxis grundsätzlich ablehnt, will sie sich nicht auf eine „Demokratisierung“ von Theorie einlassen.

Indem Arendt den Unterschied zwischen dem Verstehen der politischen Theoretikerin und der hermeneutischen Praxis politischer Akteure exponiert, hat sie das „politisch-hermeneutische Problem“ zwar in seiner Radikalität erfasst, aber keiner der Wege, die sie zur konzeptionellen Bearbeitung vorschlägt, vermag das Problem zu lösen.
So besteht die Leistung von Arendts Reflexion des Problems primär darin, diese Spannung aufrechterhalten und in einer performativen Konzeption von politischer Theorie radikalisiert zu haben, wonach die (Re-)Interpretation politischer Erfahrungen in politischen Institutionen ebenso aufgehoben ist wie in narrativen Vergegenständlichungen. Obwohl dies Arendts strikter Unterscheidung zwischen „praxis“ und „poiesis“ zunächst zuwiderläuft, wird das Politische so, wie Sigwart zu Recht betont, an die kulturelle Tätigkeit des „Herstellens“ von Bedeutungen geknüpft, gleichzeitig öffnet sie jedoch diese Manifestationen politischer Erfahrungen wieder für kreative politische Rezeptionen (386f.). Die Frage, ob der Begriff „offene politische Hermeneutik" diese Balancierung zwischen Fragilität und Stabilität angemessen zu erfassen vermag und mehr als eine allgemeine Referenz auf Pluralität beinhaltet, muss in der Arbeit mit diesem Konzept erst noch geklärt werden.

Grit Straßenberger

\section{VERGLEICH POLITISCHER SYSTEME}

Keil, Silke I. und Jan W. van Deth (Hrsg.). Deutschlands Metamorphosen - Ergebnisse des European Social Survey 2002 bis 2008, Studien zur Wahl- und Einstellungsforschung, Band 20. Baden-Baden. Nomos 2012. 436 Seiten. $69,00 €$.

Der European Social Survey (ESS) ist die bisher umfangreichste Quelle der empirischen Sozialforschung für Analysen der sozialen, politischen, gesellschaftlichen und wirtschaftlichen Entwicklung von inzwischen mehr als 30 europäischen Ländern. Der von Silke I. 
Keil und Jan W. van Deth vorgelegte Sammelband stellt mit Fokus auf Deutschland im europäischen Kontext die Ergebnisse des ESS 2002 bis 2008 vor. Herausragendes Merkmal ist ein doppelter längsschnittlicher Vergleich zwischen West- und Ostdeutschland einerseits sowie zwischen West- und Osteuropa andererseits. Durch die Verwendung gemeinsamer Daten und einheitlicher Gewichtungsvariablen erzielt der Band eine außerordentlich hohe Vergleichbarkeit. Die Datengrundlage aller Einzelbeiträge ist frei verfügbar unter http://ess.nsd.uib.no/.

Der Band gliedert sich in zwei Teile. Der erste ist mit „Kontinuität und Wandel“ überschrieben und richtet den Fokus auf längsschnittliche Analysen der stabilen beziehungsweise sich verändernden politischen und gesellschaftlichen Orientierungen der Bürger in Europa. Der zweite Teil greift unter der Überschrift „Neue Herausforderungen " ausgewählte Themen aus Querschnittsanalysen auf. Dass diese methodische Unterscheidung auch mit einer inhaltlichen Differenzierung korrespondiert, wird dabei leider nicht immer ganz deutlich.

Heiner Meulemann führt mit einem Beitrag zur Religiosität und Säkularisierung in den ersten Teil des Bandes ein. Er befasst sich mit der Tragweite der durch den Staatssozialismus erzwungenen Säkularisierung und bestätigt mit seinen Untersuchungen den Verdacht der Sonderrolle Deutschlands in Europa, dass nämlich die Unterdrückung der Kirchen in Ostdeutschland die Kirchenzugehörigkeit nachhaltiger geschwächt hat, als dies in anderen Ländern Osteuropas der Fall war.

Kai Arzheimer stellt dagegen fest, dass die Werthierarchien innerhalb Deutschlands denen Westeuropas ähneln, wäh- rend Osteuropa eine Sonderrolle einnimmt. Neu an der Erfassung der Wertorientierungen des ESS ist die Verwendung des sogenannten "Schwartz Value Inventory", welches das typischerweise verwendete InglehartInstrument ablöst. Die Vorteile liegen hierbei insbesondere in der größeren interkulturellen Vergleichbarkeit universeller Werte.

Als einer der wichtigsten deutschen Wissenschaftler der vergleichenden Wahl- und Einstellungsforschung gilt Oskar W. Gabriel, der sich in seinem Beitrag der Frage widmet, ob sich die Lebensbedingungen speziell in Ostdeutschland seit der Deutschen Einheit maßgeblich verbessert und somit auch zu einer erhöhten Lebenszufriedenheit geführt haben. Er kommt zu dem Ergebnis, dass sie sich zwar immer mehr an das Niveau im Westen angleichen, jedoch die Lebenszufriedenheit der Menschen in den neuen Bundesländern immer noch unter dem Niveau der Westdeutschen liegt.

Dieser Befund ist charakteristisch für die vorliegende Studie insgesamt und macht die Ambivalenz deutlich, mit der das Zusammenwachsen der beiden Landesteile Ost- und Westdeutschland $\mathrm{zu}$ bewerten ist. Zwar wird Gesamtdeutschland im europäischen Kontext den Ergebnissen der Studie nach mehr und mehr als ein europäisches Land wie andere auch wahrgenommen, dennoch manifestiert sich in manchen Bereichen die Sonderrolle durch die deutsch-deutsche Teilung deutlich.

Dies bestätigen auch die Ergebnisse des folgenden von Sonja Zmerli verfassten und auch für Anhänger der Politischen Theorie äußerst interessanten Artikels, der die Schwierigkeiten einer Bestimmung des Vertrauensbegriffes betont und sowohl für Ost- und Westdeutsch- 
land, als auch für Gesamtdeutschland innerhalb Europas die Wechselwirkungen in den Mittelpunkt stellt, die sich aus sozialem Vertrauen, als Voraussetzung für innergesellschaftliche Kooperation, als auch aus politischem Vertrauen, also der wesentlichen Komponente für die Legitimation demokratischer politischer Systeme, ergeben. Entgegen der von den Herausgebern verfassten Zusammenfassung der Einzelbeiträge im Einleitungsteil kommt Zmerli zu dem Ergebnis, dass sich West- und Ostdeutsche hinsichtlich des sozialen und politischen Vertrauens immer mehr angleichen und inzwischen sogar inmitten der westeuropäischen Gesellschaften angekommen sind.

Der Aufhänger für den von Silke I. Keil verfassten Beitrag ist der Bias zwischen der demokratischen Grundregel der politischen Gleichheit aller Bürger und ihrer Repräsentation im politischen System. Insbesondere ältere, ressourcenschwache Menschen beteiligen sich systematisch seltener an politischen und sozialen Prozessen als jüngere Menschen mit höherem Einkommen und höherer beruflicher Bildung. Dieser Befund wurde bereits durch viele frühere Studien erbracht, im Ergebnis neu ist hingegen, dass sich dieser Zusammenhang in Osteuropa, nicht jedoch in Ostdeutschland am deutlichsten niederschlägt. Obwohl Keil eine Differenzierung und Definition der Begrifflichkeiten anstrebt, bleiben die Fragen danach, was sie unter Gleichheit versteht, unbeantwortet. Hier hätte es dem Artikel gut getan, diese demokratietheoretische Diskussion auszusparen und stattdessen eine Definition vorzustellen, worauf sich die Untersuchungen im Detail beziehen.
Ergänzend zu Keil untersuchen Herman Schmitt und Angelika Scheuer elektorale Beteiligungsformen. Sie untersuchen die Beziehungen von Bürgern und Parteien sowohl in konsolidierten als auch postsozialistischen Demokratien. Vor dem Hintergrund der vier Standardindikatoren Volatilität, Fragmentierung, Polarisierung sowie Parteiidentifikation diskutieren die Autoren den Einfluss der Faktoren soziale Spannungslinien, Parteibindungen und Links-Rechts-Dimension auf das Wahlverhalten.

Im letzten Beitrag des ersten Teils widmen sich Jens Tenscher und Lore Hayek dem Medienkonsum. Hierfür bietet der ESS Vor- und Nachteile gleichermaßen: Zum einen liefert er Daten über allgemeinen und politikorientierten Medienkonsum sowohl im Zeit-, als auch im Ländervergleich. Zum anderen beschränken sich die Daten auf recht grobe Erhebungen zum Beispiel der Mediengattung und lassen keine Rückschlüsse auf spezifische Sender und Formate zu. Tenscher und Hayek stellen schlüssig dar, dass sich der „digital divide“ im Gegensatz zu Teilen Osteuropas innerhalb Deutschlands nahezu geschlossen hat.

Im zweiten Teil nimmt zunächst Nico Dragano den Einfluss sozialstruktureller Veränderungen auf die Gesundheit unter die Lupe, um dabei zu erstaunlichen wie gleichermaßen plausiblen Ergebnissen zu kommen: Osteuropäer nehmen seltener Medikamente ein und gehen auch seltener zum Arzt, sind aber öfter krank als Westeuropäer, während die Deutschen häufiger zum Arzt gehen als die Westeuropäer und zugleich kränker sind als diese. In der Tendenz stellt Dragano jedoch eine Angleichung der Lebensverhältnisse fest, die mit einem ähnlichen gesundheitli- 
chen Risiko- und Chancenprofil in Ost und West korrespondiert.

Daniel Fuß untersucht die Einstellungen zur Immigration auf der Individual- und Kontextebene. Sowohl für die Bundesrepublik als auch für die europäischen Länder stellt Fuß eine große Heterogenität hinsichtlich der Erfahrungen des Immigrationsgeschehens fest. Eine ablehnende Haltung gegenüber Immigration ist hauptursächlich auf Individualebene $\mathrm{zu}$ suchen und speist sich aus den subjektiv wahrgenommenen negativen Konsequenzen, insbesondere materieller Einbußen. Diese Haltung ist in Westdeutschland vergleichsweise am niedrigsten ausgeprägt, und sie nimmt insgesamt auf europäischer Ebene seit einiger Zeit sukzessive $\mathrm{ab}$.

Im Kontext der vergleichenden politischen Kulturforschung fragt Edeltraut Roller, inwieweit sich seit dem Fall der Mauer eine einheitliche deutsche beziehungsweise europäische Wohlfahrtsstaatskultur herausgebildet hat. Die Ergebnisse zeigen, dass die Ostdeutschen in Bezug auf die Extensität höhere Erwartungen an die Leistungen des Systems haben als die Westdeutschen, während sich für die europäische Ebene sagen lässt, dass sich keine homogene osteuropäische Wohlfahrtsstaatskultur als Pendant zur westeuropäischen herausgebildet hat.

Jan W. van Deth befasst sich mit der Unterstützung von Bürgertugenden. Er unterscheidet zwischen demokratischen und partizipatorischen Tugenden, wobei deutlich wird, dass soziales und politisches Engagement hinter der Unterstützung demokratischer Tugenden zurücksteht. Dieser Befund entspricht auch Ergebnissen der aktuellen Partizipationsforschung.
Zuletzt veranschaulicht Stefan Weick in seinem Beitrag die Ergebnisse der Messungen des sozialen und persönlichen Wohlbefindens auf Grundlage eines Ansatzes der Lebensqualitätsforschung. Dieser auch für Sozialpsychologen und Anhänger der Politischen Psychologie interessante Beitrag stellt den sogenannten „composite wellbeing-index" ins Zentrum, mit dem sich unter Einbeziehung von mehr als 50 Items das Wohlbefinden der Deutschen im europäischen Kontext messen lässt. Es dürfte der Gliederung des Beitrags geschuldet sein, dass dem Leser nicht auf den ersten Blick deutlich wird, wodurch sich der neue Ansatz im Vergleich zu älteren (zum Beispiel Set Point Theory) auszeichnet. Gleichwohl bieten die Ergebnisse eine hohe Anschlussfähigkeit für weitere Untersuchungen.

Obwohl der von den Herausgebern verfasste Einleitungsteil einen sehr umfänglichen Überblick über die folgenden Einzelbeiträge bietet, lassen einige Dopplungen und Widersprüche (zum Beispiel zum Wahlverhalten) den Leser stolpern. Doch insbesondere vor dem Hintergrund der letzten Bundestagssowie der bevorstehenden Europawahlen stellt der Band eine wertvolle thematische Fundgrube sowohl für Sozialwissenschaftler als auch für den interessierten Laien dar.

Sonja Clasing

Kurtz, Marcus. Latin American State

Building in Comparative Perspective. Social Foundations of Institutional Order. Cambridge. Cambridge University Press 2013. 282 Seiten. 26,99\$.

Latin American State Building in Comparative Perspective ist eine Kritik an 